\title{
Hankel forms
}

by

\section{HenRy Helson (Berkeley, CA)}

\begin{abstract}
It is an open question whether Nehari's theorem on the circle group has an analogue on the infinite-dimensional torus. In this note it is shown that if the analogue holds, then some interesting inequalities follow for certain trigonometric polynomials on the torus. We think these inequalities are false but are not able to prove that.
\end{abstract}

A Hankel form on the infinite-dimensional torus $T^{\infty}$ is a form

$$
\langle a, b\rangle=\sum_{j, k=1}^{\infty} \rho_{j k} a_{j} b_{k}
$$

where $a, b$ are square-summable, and the kernel $\left(\rho_{n}\right)(n=1,2, \ldots)$ depends on only one index, so that $j k$ in $(1)$ is a product of integers. In order for the form to be defined in $\ell^{2}, \rho$ should be square-summable; then it is bounded if there is a constant $A$ such that

$$
|\langle a, b\rangle| \leq A\|a\|_{2}\|b\|_{2}
$$

for all finitely supported sequences $a, b$. The smallest such $A$ is the bound of the form.

$T^{\infty}$ is the infinite-dimensional torus: the group of sequences

$$
x=\left(e^{i x_{1}}, e^{i x_{2}}, \ldots\right),
$$

each $x_{j}$ real, with multiplication in each component.

Denote by $\Gamma$ the group of all sequences $r=\left(n_{1}, n_{2}, \ldots\right)$ of integers terminating in 0's, with addition in each component. $\Gamma$ is dual to $T^{\infty}$ in the pairing

$$
(x, r)=\exp i \sum n_{j} x_{j} .
$$

2010 Mathematics Subject Classification: 43A15, 30B50, 15A63, 47B35.

Key words and phrases: Hankel form, Nehari's theorem, infinite-dimensional torus.

Henry Helson was born on 2 June 1927 and died on 10 January 2010. One of his two earliest papers appeared in Studia Math. 10 (1948). It was his wish to have this last paper of his appear in Studia Math., too. 
$\Gamma$ is isomorphic to a subgroup of the real line by the mapping

$$
\gamma(r)=\sum n_{j} \log p_{j},
$$

where $p_{1}=2, p_{2}=3, \ldots$ are the prime integers. We identify $r$ with the rational number

$$
\prod p_{j}^{n_{j}}
$$

so this subgroup consists of all numbers $\log r$ where $r$ is a positive rational, with addition as the group operation. This presentation of $\Gamma$ is related to Dirichlet series, and is the one we shall use.

We use the normalized Haar measure $d \sigma$ to define the Lebesgue spaces on $T^{\infty}$. A function $f$ summable on $T^{\infty}$ has Fourier coefficients $\widehat{f}(\log r)$, where $r$ ranges over the positive rational numbers. $H^{1}$ is the subspace of $L^{1}$ of all $f$ such that $\widehat{f}(\log r)=0$ unless $r$ is a positive integer. By $H_{N}$ we shall mean the space of trigonometric polynomials whose frequencies lie in the set $(\log 1, \ldots, \log N)$.

Let $\phi$ be a bounded function on $T^{\infty}$. Then the kernel $\rho_{n}=\widehat{\phi}(\log n)$ gives a Hankel form with bound $\|\phi\|_{\infty}$; the verification is straightforward. A theorem of Nehari asserts, on the integer group, that every bounded Hankel form (one with a kernel of the form $\rho_{j+k}$ ) arises from a bounded function $\phi$ on the circle, and furthermore that $\phi$ can be chosen so that $\|\phi\|_{\infty}$ equals the bound of the form. On tori of finitely many dimensions the first part of Nehari's theorem continues to hold [4]: every Hankel form arises from a bounded function, but the statement about bounds fails. The problem is whether every Hankel form on $T^{\infty}$ comes from a bounded function. The question was raised explicitly in [2]. A positive answer was given for certain forms, those of Hilbert-Schmidt type, in [3].

We suppose that the answer in general is negative, but cannot prove that. The results of this paper show that a simple and plausible statement about trigonometric polynomials in $H_{N}$ would establish this conclusion. If our plausible statement should be false, we would have a good result about $H^{1}$. The author will not be able to finish this work and so presents what he can here.

We assume what we think is false and explore the consequences.

Hypothesis. Every bounded Hankel form comes from a bounded function.

Under the Hypothesis, there is a positive number $A$ such that every form with bound $k$ is realized by a bounded function with uniform norm at most $k A$. The proof is of a familiar kind. The symbol $A$ will be reserved for this constant. The norm of a Hankel kernel $\rho$ as a bilinear form will be $\|\rho\|$. 
Our first objective is to describe a class of Hankel forms that are not of Hilbert-Schmidt type.

Let $\mathcal{A}$ be the family of non-negative functions $\rho$ on $[0, \infty)$ such that $\rho(x) x^{-1 / 2}$ decreases to 0 . It is easy to show for such $\rho$ that

$$
\sum_{n=1}^{\infty} \rho(j n) n^{-1 / 2} \leq \int_{0}^{\infty} \rho(j x) x^{-1 / 2} d x \quad(j=1,2, \ldots) .
$$

Each function $\rho$ determines a kernel: $\rho_{n}=\rho(n), n \geq 1$. The inequality leads to an estimate for the bound of the corresponding Hankel form (if the integrals in (7) are finite) by means of this result of Schur (which applies to forms that are not necessarily of Hankel type):

SCHuR's Theorem. Let $\left(\rho_{j, k}\right)$ be the kernel of a form, with each $\rho_{j, k}$ non-negative. Suppose there is a positive sequence $p$ and positive constants $c, d$ such that

$$
\begin{aligned}
& \sum_{k=1}^{\infty} \rho_{j, k} p(k) \leq c p(j) \quad(j=1,2, \ldots) \quad \text { and } \\
& \sum_{j=1}^{\infty} \rho_{j, k} p(j) \leq d p(k) \quad(k=1,2, \ldots) .
\end{aligned}
$$

Then the form is bounded with bound at most $(c d)^{1 / 2}$.

This theorem is Problem 37 of [1].

For Hankel forms, $c$ and $d$ are equal, there is only one condition, and the bound is $c$.

Take $p(n)=n^{-1 / 2}$. For each $j$ and $\rho$ in $\mathcal{A}$

$$
\sum_{k=1}^{\infty} \rho_{j k} k^{-1 / 2} \leq \int_{0}^{\infty} \rho(j x) x^{-1 / 2} d x=j^{-1 / 2} \int_{0}^{\infty} \rho(x) x^{-1 / 2} d x .
$$

By Schur's theorem, if the integral is finite, then $\rho$ defines a Hankel form with bound at most equal to the integral on the right.

Under the Hypothesis there is a constant $c$ such that for all $f$ in $H^{1}$, and all positive integers $N$ we have

$$
N^{-1 / 2}\left|\sum_{n=1}^{N} \widehat{f}(\log n)\right| \leq c\|f\|_{1} .
$$

Let $\rho$ be a decreasing sequence of positive numbers such that

$$
B=\sum_{n=1}^{\infty} \rho_{n} n^{-1 / 2}<\infty
$$

By Schur's criterion, $\rho$ defines a bounded Hankel form, and by our Hypothesis, a linear functional in $H^{1}$ with bound at most $A B$. That is, at least for 
trigonometric polynomials $f$ in $H^{1}$,

$$
\left|\sum \rho_{n} \widehat{f}(\log n)\right| \leq A B\|f\|_{1} .
$$

Choose $\rho_{n}=1$ for $n=1, \ldots, N$, and $\rho_{n}=0$ otherwise. Then $\rho$ defines a form whose bound is at most $2 N^{1 / 2}$, by (9), and $(10)$ follows.

(We can replace $\widehat{f}(\log n)$ by its modulus in 10$)$, but do not know a way to use the fact.)

For each positive integer $N$ let

$$
f_{N}=\sum_{n=1}^{N} \chi_{\log n} .
$$

This trigonometric polynomial has norm $N^{1 / 2}$ in $H^{2}$, and its norm in $H^{1}$ cannot be greater. By analogy with the Dirichlet kernel on the circle we might expect its norm in $H^{1}$ to be considerably smaller. The Hypothesis would be shown false if we could show merely that $\left\|f_{N}\right\|_{1}=o\left(N^{1 / 2}\right)$ as $N$ increases, because (10) would be violated. We think this is true but we have not found a proof. No much stronger result than $o\left(N^{1 / 2}\right)$ can hold on account of this fact (which does not depend on the Hypothesis):

TheOrem. $\left\|f_{N}\right\|_{1}$ is asymptotically at least $(N / 2 \log N)^{1 / 2}$.

We use the result in [3] that each $f$ in $H^{1}$ satisfies

$$
\left(\sum_{n=1}^{\infty}|\widehat{f}(\log n)|^{2} / d(n)\right)^{1 / 2} \leq\|f\|_{1}
$$

where $d(n)$ is the number of divisors of $n$. For $\left.f_{N}, 14\right)$ becomes

$$
\left(\sum_{n=1}^{N} 1 / d(n)\right)^{1 / 2} \leq\left\|f_{N}\right\|_{1} .
$$

If $n$ is prime, $d(n)=2$. There are about $N / \log N$ primes smaller than $N$. Counting just these terms of the sum in (15) gives our result. (Obviously the theorem can be improved by counting more terms in the sum. The $f_{N}$ are evidently very different from the Dirichlet kernel on the circle!)

The formula (14) has another interesting consequence. In the finitedimensional space $H_{N}$ the norms of $L^{1}$ and $L^{2}$ are of course equivalent; we ask what is the norm of the identity operator acting from the first to the second space? It must tend to infinity, because $H^{1}$ and $H^{2}$ are not the same spaces. $N^{1 / 2}$ is a trivial bound for the norm. But (14) shows that the bound is $O\left(N^{\epsilon}\right)$ for every positive $\epsilon$ as $N$ increases, because $d(n)$ is $O\left(n^{\epsilon}\right)$ for every positive $\epsilon$.

More generally, let $T_{p, q}$ be the identity operator from $H_{N}$ in norm $p$ to itself in norm $q$. Really $(10)$ states that $\left\|T_{1, \infty}\right\| \leq c N^{1 / 2}$. (The sum in 10) is 
simply $f(0)$ if $f$ belongs to $H_{N}$.) Now it is easy to see that $\left\|T_{2, \infty}\right\|=N^{1 / 2}$; thus the Hypothesis implies that $\left\|T_{1, \infty}\right\|$ has the same order as $\left\|T_{2, \infty}\right\|$. This seems unlikely, because $\left\|T_{1,2}\right\|$ at least tends to $\infty$ as $N$ increases(although slowly, as just shown). If the same function $g$ can nearly realize $\left\|T_{1,2}\right\|$ and $\left\|T_{2, \infty}\right\|$, then we find a contradiction. It seems likely that there is such a $g$, because given $\|g\|_{1}=1,\|g\|_{2}$ and $\|g\|_{\infty}$ are both large when $|g|$ is as far from being constant as possible in $H_{N}$. Moreover $f_{N}$ looks like a good candidate. But we cannot go further.

Appendix. Helson's paper proposes a way to prove that the classical Nehari theorem on Hankel forms does not generalize to the infinitedimensional torus. The referee of the paper pointed out a suggestive variant of Helson's approach, to which this appendix is devoted. The appendix is based on the referee's comments but was prepared by Donald Sarason, who assumed the responsibility for seeing the paper through to publication after Helson became unable to do so. Unexplained notations are as in the paper.

Helson shows that, to disprove the infinite-dimensional Nehari theorem, it suffices to prove that the estimate $\left\|f_{N}\right\|_{1}=o\left(N^{1 / 2}\right)$ fails, where

$$
f_{N}=\sum_{n=1}^{N} \chi_{\log n} .
$$

The referee introduces the functions

$$
g_{N}(t)=\sum_{n=1}^{N} n^{i t} \quad(t \in \mathbb{R}),
$$

which are partial sums of the series for $\zeta$ on the line $i \mathbb{R}$. Let

$$
\left\|g_{N}\right\|_{1}=\lim _{T \rightarrow \infty} \frac{1}{T} \int_{0}^{T}\left|g_{N}(i t)\right| d t .
$$

It is not difficult to show that $\left\|f_{N}\right\|_{1}=\left\|g_{N}\right\|_{1}$, so to complete Helson's program it suffices to prove that the estimate $\left\|g_{N}\right\|_{1}=o\left(N^{1 / 2}\right)$ fails. This transforms the question from one in harmonic analysis to one in number theory. Specialists in number theory will perhaps find the transformed question attractive.

\section{References}

[1] P. R. Halmos, A Hilbert Space Problem Book, van Nostrand, 1967.

[2] H. Helson, Dirichlet Series, Henry Helson, 2005.

[3] - Hankel forms and sums of random variables, Studia Math. 176 (2006), 85-92. 
[4] M. T. Lacey, Lectures on Nehari's theorem on the polydisk, in: Topics in Harmonic Analysis and Ergodic Theory, Contemp. Math. 444, Amer. Math. Soc., Providence, RI, 2007, 185-213.

Henry Helson

Mathematics Department

University of California

Berkeley, CA 94720-3840, U.S.A.

E-mail: hhelson@aol.com

Received October 20, 2009

To obtain a reprint of this article, contact D. Sarason (sarason@math.berkeley.edu). 